\title{
The role of mental disorders in the risk and speed of transition to alcohol use disorders among community youth
}

\author{
S. Behrendt ${ }^{1 *}$, K. Beesdo-Baum ${ }^{1}$, P. Zimmermann ${ }^{2}$, M. Höfler ${ }^{1}$, A. Perkonigg ${ }^{1}$, G. Bühringer ${ }^{1,3}$, \\ R. Lieb ${ }^{2,4}$ and H.-U. Wittchen ${ }^{1,2}$ \\ ${ }^{1}$ Institute of Clinical Psychology and Psychotherapy, Technische Universitaet Dresden, Germany \\ ${ }^{2}$ Max Planck Institute of Psychiatry, Munich, Germany \\ ${ }^{3}$ IFT Institut fuer Therapieforschung, Munich, Germany \\ ${ }^{4}$ Department of Psychology, Unit of Epidemiology and Health Psychology, University of Basel, Switzerland
}

Background. Among adolescents and young adults with DSM-IV alcohol use disorders (AUDs), there are interindividual differences in the speed of transition from initial alcohol use (AU) to AUD. AUDs are highly co-morbid with other mental disorders. The factors associated with rapid transition from first AU to AUD remain unknown and the role of mental disorders in rapid transitions is unclear. Given this background we examined (1) whether prior anxiety, mood, externalizing and non-alcohol substance use disorders are related to the risk and speed of transition from first AU to DSM-IV alcohol abuse (AA) and alcohol dependence (AD) and (2) whether early age of onset of prior mental disorders (PMDs) is a promoter of rapid transition.

Method. A total of 3021 community subjects (97.7\% lifetime AU) aged 14-24 years at baseline were followed up prospectively for up to 10 years. AU and mental disorders were assessed with the DSM-IV/M-CIDI.

Results. Among subjects with lifetime AU, several PMDs, such as specific phobia, bipolar disorder and nicotine dependence, were associated with an increased risk of AUD independent of externalizing disorders. Associations of PMDs with the speed of transition to AUDs were mostly weak and inconsistent. Only social phobia and externalizing disorders were associated with faster transitions to AD even after adjustment for other PMDs. Earlier age of onset of PMD was not associated with rapid transition.

Conclusions. Mental disorders are associated with the risk of AUD. With the possible exception of social phobia and externalizing disorders, they do not promote rapid transition, even if they occur particularly early. Future research needs to identify factors relevant to rapid transition to AUD.

Received 13 October 2009; Revised 8 June 2010; Accepted 15 June 2010

Key words: Aetiology, alcohol abuse, DSM-IV alcohol dependence, epidemiology, transition.

\section{Introduction}

In this study we investigated whether DSM-IV anxiety, mood, non-alcohol substance use disorders (DSM-IV abuse and dependence) and externalizing disorders that occur prior to DSM-IV alcohol use disorders (AUDs) are associated with an increased risk and speed of transition from first alcohol use (AU) to DSM-IV alcohol abuse (AA) and alcohol dependence (AD) in adolescence and young adulthood. In this regard, we also examined the role of early onset of prior mental disorders (PMDs) and gender.

* Address for correspondence: S. Behrendt, Ph.D., Institute of Clinical Psychology and Psychotherapy, Technische Universitaet Dresden, Chemnitzer Str. 46, D-01187 Dresden, Germany.

(Email : silke.behrendt@psychologie.tu-dresden.de)
AU is associated with the risk of serious consequences such as cardiovascular disease, unintentional injuries (Rehm et al. 2006) and AUDs. AUDs (especially AD) are disabling mental disorders (Hasin et al. 2007) and may occur as early as in adolescence and young adulthood (Bonomo et al. 2004; Grant et al. 2004a; Nelson \& Wittchen, 1998). AD with onset in adolescence is especially malignant (Hingson et al. 2006). AUDs are highly co-morbid with mood, anxiety, externalizing and other substance use disorders. Co-morbidity is more frequent in younger adults (Burns \& Teesson, 2002; Kendler et al. 2003; Grant et al. 2004b; Hasin et al. 2007).

Mental disorders and their symptoms are risk factors for AUDs and problematic AU (Kushner et al. 1999; Crum \& Pratt, 2001; Zimmermann et al. 2003; Goodwin et al. 2004; Haynes et al. 2005). For example, 
social phobia and pronounced depressive symptoms predicted AD (Gilman \& Abraham, 2001; Crum et al. 2008a; Buckner \& Turner, 2009). Externalizing disorders are well-documented risk factors for AUDs (Elkins et al. 2007). However, few studies on this topic fulfill all of the following criteria: inclusion of young adolescents, a long follow-up interval and a wide range of DSM-IV diagnoses considered as predictors and control variables (Buckner et al. 2008a). Therefore, we investigate various DSM-IV PMDs as risk factors for DSM-IV AUDs with thorough adjustment for covariates in a 10-year prospective epidemiological study including young adolescents.

We hypothesized that an excess risk of AUD exists for especially early PMD onset. Earlier PMDs show greater severity and co-morbidity, poorer health outcomes and problematic coping styles (Beesdo et al. 2007; Keenan-Miller et al. 2007; Hammen et al. 2008; Sansone \& Sansone, 2009). Early-onset PMDs predict problematic substance use behaviors that are related to AUDs (DeWit et al. 2000; Bonomo et al. 2004; Crum et al. 2008b; Sihvola et al. 2008). Major depression and social phobia also occurred earlier in subjects with secondary AUD than in subjects without AUD (Grant et al. 1996; Buckner et al. 2008b).

It is also of interest to determine whether PMDs play a role in the speed of transition to AUDs. The substance-specific latency between first use and onset of a substance use disorder is longer for alcohol than for cannabis and cocaine (Wagner \& Anthony, 2002; Behrendt et al. 2009). It can span more than 10 years (DeWit et al. 2000) with considerable inter-individual variation. Some adolescents develop an AUD in the first 2 years after first AU (Wittchen et al. 2008). Information on the promoters of such rapid transitions would improve the understanding of individual differences in AUD development and help to identify subjects who need timely interventions (Wittchen et al. 2008) and have poorer AD prognoses (Hingson et al. 2006). However, factors characterizing these subjects remain understudied. Because of lower self-efficacy (John et al. 2004), higher impulsivity (Kliegel et al. 2006), and instrumental AU (Thomas et al. 2003) in mental disorders, PMDs may lead to reduced control over AU. Therefore, we hypothesized that any and early PMDs are associated with faster transitions to AUDs.

Although gender differences in AUDs (Grant et al. 2004a), mood, anxiety and externalizing disorders (Blazer et al. 1994; Wittchen et al. 1998b; Kendler et al. 2003; Wilhelm et al. 2003) are well documented, the role of gender in the relationship between PMD and the speed of transition to AUD remains unclear. Female gender has been linked with faster transitions to alcohol problems (Randall et al. 1999), but more recent studies found no or little evidence for a faster AUD development in women (Wagner \& Anthony, 2007; Wittchen et al. 2008). We hypothesized that there are no gender differences in the speed of transition to AUD.

Given this background we examined whether (1) PMDs are associated with a higher risk of AUDs and a higher speed of transition from first AU to AUD, (2) associations with speed of transition differ by gender and (3) early PMD onset is associated with the risk and speed of transition.

\section{Method}

\section{Study sample and design}

The Early Developmental Stages of Psychopathology (EDSP) study is a prospective longitudinal community study on the prevalence, course, vulnerabilities and risk factors of substance use and substance use disorders in adolescence and early adulthood. The study includes a baseline (T0; conducted in 1995) and three follow-up assessments (T1, T2, T3) (Wittchen et al. 1998c; Lieb et al. 2000 ; Beesdo et al. 2010).

The study sample consisted of 3021 Germanspeaking subjects (1533 males, 1488 females) aged 1424 years at baseline. The sample was drawn randomly from government registries in metropolitan Munich, Germany. As the study focused on early developmental stages of psychopathology, individuals aged 14-15 years were sampled at twice the probability of those aged 16-21 years, who were sampled at twice the probability of those aged 22-24 years. The followup examinations were carried out approximately 1.6 years ( 11 , median interval since baseline), 3.5 years (T2) and 8.2 years (T3) later. At T1, only the younger cohort of subjects aged 14-17 years at baseline was assessed $(n=1228)$. Response rates were $70.9 \%$ at T0 $(n=3021), 84.3 \%(n=2548)$ at T2 and $73.2 \%(n=2210)$ at T3. At T3, the age range was $21-34$ years. Further detailed information on the study can be found elsewhere (Wittchen et al. 1998c; Lieb et al. 2000).

Participants were asked whether they were willing to answer questions on illegal substances truthfully (commitment probe). A total of 142 subjects who declined at one or more waves were excluded from all analyses with variables concerning illegal substances.

Any AU, regular AU (at least weekly) or hazardous AU ( $\geqslant 20 \mathrm{~g}$ of ethanol for women or $\geqslant 40 \mathrm{~g}$ of ethanol for men almost every day) at T0 did not predict dropout at any follow-up, with one exception: in the younger cohort, regular AU at T0 predicted drop-out at the first but not at any other follow-up (if subjects did not participate in T1, the T0-T2 interval was covered in the $\mathrm{T} 2$ assessment). 
The cumulative incidence rate up to T3 was $97.7 \%$ for $\mathrm{AU}(n=2929), 24.7 \%$ for AA $(n=741)$ and $11.0 \%$ for $\operatorname{AD}(n=327)$.

\section{Diagnostic assessment}

At all assessment waves, participants were interviewed face to face with the computer-assisted, fully standardized Munich-Composite International Diagnostic Interview (DIA-X/M-CIDI; Wittchen \& Pfister, 1997; Wittchen et al. 1998a), an updated version of the World Health Organization (WHO) CIDI (Wittchen \& Semmler, 1990). The lifetime version was used at baseline; a follow-up interval version was used at the subsequent waves. With the DIA-X/MCIDI it is possible to assess symptoms, syndromes and diagnoses of 48 mental disorders, along with information about onset, severity and impairment. For the diagnoses presented here, the computerized M-CIDI/ DSM-IV algorithms were applied. The DIA-X/M-CIDI is supplemented by a respondent's booklet including symptom lists and cognitive aids to help the respondent with answering symptom questions. The testretest reliability and validity of the DIA-X/M-CIDI diagnoses have been established (Lachner et al. 1998; Reed et al. 1998; Wittchen et al. 1998a). Interviewers, most of whom were clinical psychologists, received intensive training on the DIA-X/M-CIDI, followed by monitored practice interviews at baseline and booster sessions before each subsequent wave. Further information has been provided elsewhere (Wittchen et al. 1998c; Lieb et al. 2000).

\section{Assessment of AU and AUD}

AU and AUD were assessed with the DIA-X/M-CIDI section on AU, which begins with questions on quantity, frequency, age of onset and age of recency of use. For the assessment of diagnostic criteria, at least minimal AU was required; defined as (1) AU at least three times a week, or more than three 'standard drinks' per drinking day, in subjects who had drunk on more than 12 occasions in at least 1 year of their lives (applied for AD) or (2) AU on at least 13 occasions in a 12-month period (for AA). AD was also assessed in subjects who met the minimal AU criteria in shorter periods. The following AU levels were considered here: any use, DSM-IV dependence and DSMIV abuse (non-hierarchical).

\section{Statistical analysis}

To account for different sampling probabilities at baseline according to age, and response rates at baseline varying over age, gender and geographic region, data were weighted. The Stata Software package version 11.0 (StataCorp, 2009) was used for all calculations and to compute robust variances, confidence intervals (CIs) and $p$ values (by applying the HuberWhite sandwich matrix) required when basing analyses on weighted data (Royall, 1986). Cumulative lifetime incidence was generated using the last observation carried forward (LOCF) method, that is the information obtained until the last available assessment was taken into account. This enabled us to use information from subjects who had dropped out of the study during the assessments. According to CIDI conventions, age of AUD onset was defined as age at first AUD symptom.

The Kaplan-Meier (Therneau \& Grambsch, 2000) estimator was used to estimate the age-dependent cumulative lifetime incidence of AU and mental disorders. Cox regressions were applied for assessing overall differences in risk of transition from first AU to AUD over time (time scale=years from first AU to AUD) across PMD status. Covariates (factors of interest and control variables) were entered into the Cox regression analysis as time-dependent covariates to ensure that PMD had occurred prior to AUD [age of onset of PMD $<t, t=1,2, \ldots$, minimum (age of onset AUD - age of onset AU, age at last assessment - age of onset $\mathrm{AU})]$. In the Cox regression, cases with $\mathrm{AU}$ and AUD onset within the same 12-month period (i.e. length of transition $=0$ years) are excluded automatically in Stata (StataCorp, 2009). To prevent this, we shifted the time scale by 1 year upwards, by replacing 0 years by 1 year, 1 year by 2 years, and so on.

Different curves according to birth cohort and gender were allowed for by fitting stratified Cox regressions (Therneau \& Grambsch, 2000). Schoenfeld residuals were used to test whether group differences varied over time (Therneau \& Grambsch, 2000). When necessary, the interaction term covariate $\times$ number of years since AU was added to the model, to improve the model fit and to assess how strongly the hazard ratios (HRs) varied over time. Here, the model-based time-dependent HR is given by: $\mathrm{HR}(t)=\mathrm{HR}$ (main effect of covariate) $\times \mathrm{HR}$ (interaction effect of covariate) ${ }^{t}$, where $t$ is the number of years since onset of AU.

For the Cox regression analysis, we used data from subjects with lifetime AU $(n=2929)$. Few subjects had reported AU, AUD or mental disorders other than AUD, but had not provided the respective age of onset information. Data from these subjects were excluded from the survival analyses ${ }^{1} \uparrow$ To assess whether early PMD onset was associated with a higher speed of transition to AUD we used the dimensional age of onset of the respective PMD, that is we determined

\footnotetext{
$\dagger$ The notes appear after the main text.
} 
Table 1. Sequence of alcohol use, DSM-IV alcohol use disorders (AUDs) and co-morbid mental disorders (T0-T3; $n=3021)^{\mathrm{a}}$

\begin{tabular}{|c|c|c|c|c|c|c|c|c|c|}
\hline & \multirow[b]{3}{*}{$n^{\text {ag }}$} & \multirow[b]{3}{*}{$\% w^{b}$} & \multicolumn{6}{|c|}{$\begin{array}{l}\text { Onset of diagnosis in relation to alcohol use (AU), } \\
\text { alcohol abuse or alcohol dependence (AD) }\end{array}$} & \multirow{3}{*}{$\begin{array}{l}\text { Total } \\
n^{\mathrm{f}}\end{array}$} \\
\hline & & & \multicolumn{2}{|c|}{ Primary } & \multicolumn{2}{|c|}{ Same year } & \multicolumn{2}{|c|}{ Secondary } & \\
\hline & & & $n$ & $\% W^{c}$ & $n$ & $\% w^{c}$ & $n$ & $\% w^{c}$ & \\
\hline \multicolumn{10}{|l|}{ Occurring alcohol use } \\
\hline Any affective disorder ${ }^{\mathrm{i}}$ & 885 & 98.59 & 169 & 17.88 & 63 & 5.94 & 636 & 76.18 & 868 \\
\hline Major depression & 623 & 98.67 & 82 & 12.77 & 36 & 4.88 & 493 & 82.35 & 611 \\
\hline Dysthymia & 148 & 98.39 & 38 & 23.38 & 8 & 5.36 & 98 & 71.25 & 144 \\
\hline Bipolar disorder (I or II) & 123 & 97.09 & 32 & 24.12 & 16 & 11.37 & 73 & 64.51 & 121 \\
\hline Any anxiety disorder ${ }^{\mathrm{i}}$ & 951 & 97.66 & 598 & 64.18 & 49 & 4.95 & 248 & 30.87 & 895 \\
\hline Specific phobia & 498 & 97.69 & 374 & 77.31 & 19 & 3.98 & 75 & 18.71 & 468 \\
\hline Social phobia & 209 & 94.40 & 105 & 53.27 & 19 & 8.68 & 71 & 38.04 & 195 \\
\hline Panic attacks & 283 & 99.42 & 55 & 17.98 & 18 & 6.77 & 203 & 75.25 & 276 \\
\hline Somatoform disorders & 1055 & 98.12 & 477 & 44.52 & 91 & 8.83 & 460 & 46.65 & 1028 \\
\hline Any substance use disorder ${ }^{\mathrm{i}}$ & 984 & 99.81 & 34 & 3.11 & 64 & 6.28 & 837 & 90.61 & 935 \\
\hline Nicotine dependence & 847 & 99.94 & 35 & 3.58 & 61 & 6.63 & 741 & 89.79 & 837 \\
\hline Cannabis abuse $^{\text {de }}$ & 304 & 99.51 & 6 & 1.44 & 9 & 2.04 & 286 & 96.52 & 301 \\
\hline Externalizing disorder ${ }^{h}$ & 360 & 98.52 & 189 & 53.59 & 59 & 16.98 & 107 & 29.42 & 355 \\
\hline \multicolumn{10}{|l|}{ Co-morbid alcohol abuse $\mathrm{e}^{\mathrm{e}}$} \\
\hline Any affective disorder ${ }^{\mathrm{i}}$ & 885 & $28.12^{*}$ & 131 & 50.16 & 27 & 9.83 & 104 & 40.01 & 262 \\
\hline Major depression & 623 & $25.98^{*}$ & 65 & 38.31 & 15 & 9.17 & 88 & 52.52 & 168 \\
\hline Dysthymia & 148 & $31.07^{*}$ & 24 & 47.78 & 5 & 11.14 & 16 & 41.07 & 45 \\
\hline Bipolar disorder (I or II) & 123 & $38.47^{*}$ & 31 & 59.55 & 7 & 13.09 & 14 & 27.36 & 52 \\
\hline Any anxiety disorder ${ }^{\mathrm{i}}$ & 951 & $25.27^{*}$ & 188 & 80.71 & 7 & 2.62 & 38 & 16.66 & 233 \\
\hline Specific phobia & 498 & $24.48^{*}$ & 106 & 92.68 & 2 & 1.24 & 6 & 6.07 & 114 \\
\hline Social phobia & 209 & $31.19^{*}$ & 49 & 74.42 & 1 & 2.85 & 13 & 22.73 & 63 \\
\hline Panic attacks & 283 & $32.96^{*}$ & 35 & 40.15 & 9 & 8.84 & 47 & 51.01 & 91 \\
\hline Somatoform disorders & 1055 & $23.67^{*}$ & 177 & 67.61 & 21 & 8.10 & 54 & 24.29 & 252 \\
\hline Any substance use disorder ${ }^{\mathrm{i}}$ & 984 & $41.40^{*}$ & 119 & 30.72 & 85 & 19.21 & 193 & 50.07 & 397 \\
\hline Nicotine dependence & 847 & $38.66^{*}$ & 106 & 32.02 & 70 & 18.92 & 155 & 49.06 & 331 \\
\hline Cannabis abuse $^{\text {de }}$ & 304 & $64.17^{*}$ & 30 & 15.87 & 30 & 15.03 & 187 & 69.10 & 187 \\
\hline Externalizing disorder ${ }^{\mathrm{h}}$ & 360 & $54.78^{*}$ & 191 & 96.06 & 8 & 2.92 & 2 & 1.03 & 201 \\
\hline \multicolumn{10}{|l|}{ Co-morbid alcohol dependence } \\
\hline Any affective disorder ${ }^{\mathrm{i}}$ & 885 & $14.48^{*}$ & 83 & 60.27 & 15 & 12.01 & 39 & 27.72 & 137 \\
\hline Major depression & 623 & $12.99 *$ & 40 & 46.74 & 11 & 11.18 & 36 & 42.08 & 87 \\
\hline Dysthymia & 148 & $19.83^{*}$ & 18 & 56.55 & 3 & 10.44 & 9 & 33.01 & 30 \\
\hline Bipolar disorder (I or II) & 123 & $23.56^{*}$ & 20 & 69.30 & 1 & 3.19 & 9 & 27.52 & 30 \\
\hline Any anxiety disorder ${ }^{\mathrm{i}}$ & 951 & $14.11^{*}$ & 115 & 85.87 & 3 & 3.09 & 14 & 11.04 & 132 \\
\hline Specific phobia & 498 & $14.96^{*}$ & 68 & 90.55 & 1 & 4.34 & 4 & 5.11 & 73 \\
\hline Social phobia & 209 & $18.74^{*}$ & 27 & 64.56 & 2 & 7.16 & 10 & 28.28 & 39 \\
\hline Panic attacks & 283 & $19.72^{*}$ & 23 & 39.51 & 8 & 14.94 & 22 & 45.55 & 53 \\
\hline Somatoform disorders & 1055 & $11.54^{*}$ & 86 & 63.97 & 10 & 7.79 & 27 & 28.24 & 123 \\
\hline Any substance use disorder ${ }^{i}$ & 984 & $23.52^{*}$ & 97 & 42.79 & 46 & 21.98 & 68 & 35.22 & 211 \\
\hline Nicotine dependence & 847 & $22.95^{*}$ & 87 & 45.31 & 40 & 21.80 & 56 & 32.98 & 183 \\
\hline Cannabis abuse $^{\text {de }}$ & 304 & $37.01^{*}$ & 26 & 24.68 & 16 & 12.97 & 55 & 62.34 & 97 \\
\hline Externalizing disorder ${ }^{\mathrm{h}}$ & 360 & $28.90^{*}$ & 93 & 91.49 & 7 & 8.51 & 0 & 0.00 & 100 \\
\hline
\end{tabular}

${ }^{a}$ Based on cumulative incidence; T0-T3; $n=3021$.

${ }^{\mathrm{b}}$ Weighted percentage of AU and AUD among subjects with the respective mental disorder.

${ }^{\mathrm{c}}$ Weighted row percentages.

${ }^{\mathrm{d}} n=142$ excluded (unwilling to answer drug questions truthfully). 
whether higher (compared to lower) age of onset was associated with the speed of transition. Subjects with PMD onset after or in the same year as AUD onset were excluded from this analysis.

First, the Cox regression analysis was conducted with adjustment for age and gender (model I). In model II, we additionally adjusted for other PMDs: if the covariate of interest was a mood disorder, we adjusted for any anxiety disorder and non-alcohol substance use disorders (nicotine dependence, cannabis abuse or dependence, abuse or dependence of illegal drugs other than cannabis). If the covariate of interest was an anxiety disorder, we adjusted for any mood disorder and non-alcohol substance use disorders. When the covariate of interest was a non-alcohol substance use disorder, we adjusted for all other nonalcohol substance use disorders, any anxiety and any mood disorder. With externalizing disorders as covariate, we adjusted for any mood, any anxiety and all non-alcohol substance use disorders. For anxiety disorders that were significant predictors in model II, we repeated model II with additional adjustment (model IIA) for selected other anxiety disorders (other anxiety disorders were selected as covariates only if they were significant predictors in model II) and for a category of those anxiety disorders that are otherwise not considered as covariates here because of insufficient power (see next section). In model III, we repeated model II with additional adjustment for externalizing disorders. Statistical power did not permit differentiation between substance use disorders related to illegal drugs other than cannabis.

\section{Covariates}

Covariates (PMDs) were calculated from their cumulative lifetime status and the respective age of onset variable for: any mood, any anxiety and any nonalcohol substance use disorder ${ }^{2}$, major depression, dysthymia, bipolar disorder (bipolar I and bipolar II disorder), specific phobia, social phobia, nicotine dependence, cannabis abuse (non-hierarchical) and somatoform disorders (any DSM-IV somatoform disorder or somatoform/dissociative syndrome including SSI 4/6). For phobias, impairment was assessed but only requested for DSM-IV diagnosis after age 17 because of the possibly limited reliability of impairment reports in young respondents and because these disorders occur particularly early (Wittchen et al. 1999a,b). DSM-IV panic attacks were considered and are subsequently listed among the anxiety disorders but not included in the any anxiety disorder variable. Diagnosis of externalizing disorders (conduct or antisocial personality disorder) was obtained from parental reports on conduct disorder at T1 and participants' reports on both disorders at T2 resulting in information on 2638 subjects. Age of onset of antisocial personality disorder was set to age 13 by convention $^{3}$.

\section{Results}

\section{Co-morbidity and temporal sequence}

Table 1 shows that rates of lifetime AU in subjects with lifetime mental disorders were comparable to those in the entire sample. The risk of lifetime AUD was elevated for all considered lifetime disorders (all $p$ values $<0.05$ ). In the case of lifetime co-morbidity, onset of specific mental disorders occurred prior to AU in over $50 \%$ of subjects with specific phobia, social phobia and externalizing disorders, but secondary to AU for 64.5$96.5 \%$ of cases of all other mental disorders except somatoform disorders. Other mental disorders mainly occurred primary to AA (except major depression, dysthymia, panic attacks and substance use disorders) and to AD (except substance use disorders, major depression and panic attacks).

\section{PMD and the risk and speed of transition to AUD}

Model I: adjusted for age and gender. Table 2 shows that all mood and anxiety disorders, cannabis abuse, nicotine dependence, somatoform and externalizing disorders were associated with a higher risk for AA and

\footnotetext{
(Table 1 footnote continued)

${ }^{\mathrm{e}}$ Hierarchy rule not applied.

${ }^{\mathrm{f}}$ Number of co-morbid cases; only cases who provided age of onset information were considered.

${ }^{\mathrm{g}}$ Total number of cases with the respective diagnosis in the sample (irrespective of alcohol use or alcohol use disorder).

${ }^{\mathrm{h}}$ Either conduct disorder or antisocial personality disorder; based on information from 2638 subjects at T1 and T2.

${ }^{\mathrm{i}}$ Any anxiety disorder: panic disorder, agoraphobia without history of panic disorder, generalized anxiety disorder, obsessive-compulsive disorder (OCD), post-traumatic stress disorder (PTSD), specific and social phobia; any mood disorder: major depression, dysthymia, bipolar disorder I or II; any non-alcohol substance use disorder: nicotine dependence or any illegal substance use disorder.

* Higher risk of lifetime alcohol abuse or dependence (odds ratio adjusted for gender and age at last observation significant with $p<0.05$; table with odds ratios and $95 \%$ confidence intervals available upon request).
} 
Table 2. The risk of DSM-IV alcohol abuse and dependence by prior mental disorders (PMDs): overall difference in models I-III ${ }^{\mathrm{a}}$

\begin{tabular}{|c|c|c|c|c|c|c|c|c|}
\hline & \multicolumn{4}{|c|}{ Alcohol abuse (AA) } & \multicolumn{4}{|c|}{ Alcohol dependence (AD) } \\
\hline & HR & $95 \%$ CI & $p$ & $\begin{array}{l}\text { Probability }>\chi^{2} \\
(p \text { value })^{\mathrm{b}}\end{array}$ & HR & $95 \% \mathrm{CI}$ & $p$ & $\begin{array}{l}\text { Probability }>\chi^{2} \\
(p \text { value })^{\mathrm{b}}\end{array}$ \\
\hline \multicolumn{9}{|l|}{ Model I ${ }^{\mathrm{a}}$} \\
\hline Any affective disorderg & 1.67 & $1.3-2.0$ & $<0.001$ & 0.08 & 2.74 & $2.0-3.6$ & $<0.001$ & 0.30 \\
\hline Major depression & 1.33 & $1.05-1.7$ & 0.016 & 0.48 & 1.93 & $1.3-2.7$ & $<0.001$ & 0.43 \\
\hline Dysthymia & 1.75 & $1.2-2.5$ & 0.002 & 0.58 & N.A. ${ }^{c}$ & & & \\
\hline Bipolar disorder & 2.03 & $1.3-3.0$ & $<0.001$ & 0.19 & 2.99 & $1.8-4.9$ & $<0.001$ & 0.58 \\
\hline Any anxiety disorderg & 1.44 & $1.2-1.7$ & $<0.001$ & 0.89 & 2.44 & $1.9-3.1$ & $<0.001$ & 0.91 \\
\hline Specific phobia & 1.38 & $1.1-1.7$ & 0.005 & 0.81 & 2.40 & $1.7-3.3$ & $<0.001$ & 0.59 \\
\hline Social phobia & 1.74 & $1.2-2.4$ & $<0.001$ & 0.32 & 2.31 & $1.5-3.4$ & $<0.001$ & 0.13 \\
\hline Panic attacks & 1.80 & $1.3-2.4$ & $<0.001$ & 0.62 & 2.69 & $1.8-4.0$ & $<0.001$ & 0.22 \\
\hline Somatoform disorders & 1.41 & $1.1-1.7$ & $<0.001$ & 0.74 & 1.53 & $1.1-2.0$ & 0.003 & 0.30 \\
\hline Any substance use disorderg & 2.19 & $1.8-2.6$ & $<0.001$ & 0.58 & 4.20 & $3.2-5.5$ & $<0.001$ & 0.21 \\
\hline Nicotine dependence & 1.94 & $1.6-2.3$ & $<0.001$ & 0.18 & 4.11 & $3.1-5.4$ & $<0.001$ & 0.28 \\
\hline Cannabis abuse $\mathrm{de}^{\mathrm{de}}$ & 2.72 & $2.0-3.7$ & $<0.001$ & 0.56 & 2.66 & $1.8-3.9$ & $<0.001$ & 0.98 \\
\hline Externalizing disorder ${ }^{\mathrm{f}}$ & 2.46 & $2.0-3.0$ & $<0.001$ & 0.92 & 3.02 & $2.2-4.0$ & $<0.001$ & 0.00 \\
\hline \multicolumn{9}{|l|}{ Model II ${ }^{\text {ad }}$} \\
\hline Any affective disorderg & 1.39 & $1.1-1.7$ & 0.001 & 0.32 & 1.75 & $1.2-2.4$ & 0.001 & 0.37 \\
\hline Major depression & 1.12 & $0.8-1.4$ & 0.324 & 0.86 & 1.27 & $0.8-1.8$ & 0.197 & 0.51 \\
\hline Dysthymia & 1.29 & $0.8-1.9$ & 0.181 & 0.54 & N.A. ${ }^{c}$ & & & \\
\hline Bipolar disorder & 1.76 & $1.1-2.6$ & 0.004 & 0.30 & 1.84 & $1.1-3.0$ & 0.013 & 0.27 \\
\hline Any anxiety disorderg & 1.22 & $1.01-1.5$ & 0.039 & 0.85 & 1.96 & $1.4-2.6$ & $<0.001$ & 0.38 \\
\hline Specific phobia & 1.21 & $0.9-1.5$ & 0.106 & 0.67 & 1.93 & $1.3-2.7$ & $<0.001$ & 0.68 \\
\hline Social phobia & 1.39 & $1.00-1.9$ & 0.044 & 0.43 & 1.74 & $1.1-2.6$ & 0.006 & 0.24 \\
\hline Panic attacks & 1.44 & $1.05-2.0$ & 0.023 & 0.78 & 1.55 & $1.02-2.3$ & 0.036 & 0.22 \\
\hline Somatoform disorders & 1.18 & $0.9-1.4$ & 0.091 & 0.38 & 1.10 & $0.8-1.5$ & 0.505 & 0.16 \\
\hline Any substance use disorderg & 2.02 & $1.6-2.4$ & $<0.001$ & 0.75 & 3.50 & $2.6-4.6$ & $<0.001$ & 0.30 \\
\hline Nicotine dependence & 1.58 & $1.2-1.9$ & $<0.001$ & 0.35 & 2.96 & $2.1-4.0$ & $<0.001$ & 0.37 \\
\hline Cannabis abuse $\mathrm{de}^{\mathrm{de}}$ & 2.20 & $1.6-3.0$ & $<0.001$ & 0.80 & 1.37 & $0.9-2.1$ & 0.130 & 0.60 \\
\hline Externalizing disorder ${ }^{\mathrm{f}}$ & 2.11 & $1.7-2.6$ & $<0.001$ & 0.82 & 2.19 & $1.6-3.0$ & $<0.001$ & 0.01 \\
\hline \multicolumn{9}{|l|}{ Model IIA ${ }^{\mathrm{a}}$} \\
\hline Specific phobia & & & & & 1.85 & $1.3-2.6$ & $<0.001$ & 0.50 \\
\hline Social phobia & 1.33 & $0.9-1.9$ & 0.083 & 0.32 & 1.41 & $0.9-2.2$ & 0.116 & 0.36 \\
\hline Panic attacks & 1.38 & $0.9-2.0$ & 0.080 & 0.98 & 1.11 & $0.6-1.9$ & 0.694 & 0.43 \\
\hline \multicolumn{9}{|l|}{ Model III ${ }^{\text {ad }}$} \\
\hline Any affective disorderg & 1.41 & $1.1-1.7$ & 0.001 & 0.93 & 1.78 & $1.2-2.5$ & 0.001 & 0.81 \\
\hline Major depression & 1.17 & $0.9-1.5$ & 0.208 & 0.55 & 1.29 & $0.8-2.0$ & 0.226 & 0.36 \\
\hline Dysthymia & 1.35 & $0.8-2.1$ & 0.164 & 0.38 & N.A. ${ }^{c}$ & & & \\
\hline Bipolar disorder & 1.67 & $1.1-2.4$ & 0.007 & 0.76 & 2.04 & $1.2-3.3$ & 0.003 & 0.14 \\
\hline Any anxiety disorderg & 1.07 & $0.8-1.3$ & 0.472 & 0.46 & 1.59 & $1.1-2.2$ & 0.003 & 0.72 \\
\hline Specific phobia & 1.07 & $0.8-1.4$ & 0.574 & 0.29 & 1.62 & $1.1-2.3$ & 0.007 & 0.73 \\
\hline Social phobia & 1.26 & $0.8-1.8$ & 0.187 & 0.52 & 1.39 & $0.8-2.2$ & 0.155 & 0.09 \\
\hline Panic attacks & 1.34 & $0.9-1.9$ & 0.084 & 0.93 & 1.35 & $0.8-2.1$ & 0.158 & 0.40 \\
\hline Somatoform disorders & 1.14 & $0.9-1.4$ & 0.210 & 0.56 & 1.13 & $0.8-1.5$ & 0.414 & 0.49 \\
\hline Any substance use disorderg & 1.64 & $1.3-2.0$ & $<0.001$ & 0.52 & 2.95 & $2.1-4.1$ & $<0.001$ & 0.96 \\
\hline Nicotine dependence & 1.36 & $1.09-1.7$ & 0.006 & 0.56 & 2.62 & $1.8-3.7$ & $<0.001$ & 0.65 \\
\hline Cannabis abuse $\mathrm{de}^{\mathrm{de}}$ & 1.80 & $1.2-2.6$ & 0.001 & 0.60 & 1.10 & $0.6-1.8$ & 0.681 & 0.61 \\
\hline
\end{tabular}

HR, Hazard ratio ; CI, confidence interval; N.A., not applicable.

${ }^{a}$ Model I: adjusted for gender and age at last observation. Model II: additionally adjusted for substance use disorders (nicotine dependence, cannabis abuse or dependence, abuse or dependence of illegal drugs other than cannabis), any anxiety disorder (not if the covariate of interest was an anxiety disorder), and any mood disorder (not if the covariate of interest was a mood disorder). When the covariate of interest was a substance use disorder, the specific analysis was not adjusted for this 
AD (comparison group in models I-III were subjects without the respective PMD). Two significant interactions with time (not shown in Table 2) were found: social phobia was associated with a lower speed of transition to AD (main effect HR 1.19, interaction effect HR 1.14, 95\% CI 1.02-1.27, $p=0.019)$. Externalizing disorders were associated with a higher speed of transition to $\mathrm{AD}$ (main effect $\mathrm{HR} 4.69$, interaction effect HR $0.89,95 \%$ CI $0.82-0.98, p=0.018)$.

Model II: additional adjustment for other PMD. Bipolar disorder, social phobia, panic attacks, nicotine dependence, cannabis abuse and externalizing disorders were associated with a higher risk of AA. AD was predicted by bipolar disorder, specific phobia, social phobia, panic attacks, nicotine dependence and externalizing disorders. Surprisingly, in model II, social phobia was associated with a higher speed of transition to AD (main effect HR 0.95, interaction effect HR $1.12,95 \%$ CI 1.008-1.26, $p=0.035$ ), as were externalizing disorders (main effect HR 3.25, interaction effect HR $0.90,95 \%$ CI $0.83-0.98, p=0.027)$.

Model IIA: adjustment for selected anxiety disorders. In this model, all associations were adjusted for rare anxiety disorders and for those particular anxiety disorders that were significant predictors in model II. Social phobia did not predict AA (additional covariate: panic attacks). Panic attacks did not predict AA (additional covariate: social phobia). Specific phobia predicted AD (additional covariates: social phobia, panic attacks). Social phobia was not associated with the risk but with a higher speed of transition to AD (main effect HR 0.79, interaction effect HR 1.12, 95\% CI 1.002-1.26, $p=0.045$; additional covariates: specific phobia, panic attacks). Panic attacks did not predict AD (additional covariates: specific and social phobia).
Model III: additional adjustment for externalizing disorders. In this model, bipolar disorder, cannabis abuse and nicotine dependence were associated with a higher risk of AA. AD was predicted by bipolar disorder, specific phobia and nicotine dependence. Social phobia was associated with a higher speed of transition to AD (main effect HR 0.69, interaction effect HR $1.14,95 \%$ CI $1.009-1.30, p=0.036)$.

\section{Gender differences}

To assess whether associations between PMD and the speed of transition to AUD differed by gender, we added the interaction term PMD $\times$ gender $\times$ time to the model. The only significant interaction was found for dysthymia $\times$ gender $\times$ time (AA as outcome, $p=0.015$ ). In the male and female subgroups, the results on the association between dysthymia $\times$ time and AA indicated a trend towards a faster transition to AA in women with dysthymia (main effect HR 2.19, interaction effect HR $0.84,95 \%$ CI 0.70-1.02, $p=0.093$ ) and a trend towards a slower transition to AA in men (main effect HR 1.91, interaction effect HR 1.05, 95\% CI 0.92 $1.18, p=0.424)$, but the results were not significant.

\section{Earlier PMD onset}

Earlier age of onset of major depression, dysthymia, specific phobia and cannabis abuse was associated with a higher risk of AA. In model II, this was found for major depression and cannabis abuse. Table 3 shows that earlier onset of major depression, specific phobia and nicotine dependence was associated with a higher risk of $\mathrm{AD}$ in both models.

In model I, later age of onset of panic attacks was marginally associated with a faster transition to AA (main effect HR 0.73, interaction effect HR 1.07, 95\% CI 1.01-1.13, $p=0.011$ ) and AD (main effect HR 0.63,

(Table 2 footnote continued)

particular, but for all other substance use disorders. When the covariate of interest was an externalizing disorder, the analysis was adjusted for any mood, any anxiety and all non-alcohol substance use disorders. Model IIA: as model II but with additional adjustment for anxiety disorders that predicted the respective alcohol use disorder (AUD) in model II and for an aggregated variable including generalized anxiety disorder, obsessive-compulsive disorder (OCD), post-traumatic stress disorder (PTSD), agoraphobia without history of panic disorder and panic disorder. Model III: as model II with additional adjustment for externalizing disorders.

b Schoenfeld Residual Test with $p<0.05$ indicates that HRs depend on time.

${ }^{\mathrm{c}}$ Not applicable because of insufficient statistical power.

$\mathrm{d}_{n=142}$ excluded (unwilling to answer drug questions truthfully).

e Hierarchy rule not applied.

${ }^{\mathrm{f}}$ Either conduct disorder or antisocial personality disorder; information available from T1 and T2 ( $\left.n=2638\right)$.

g Any anxiety disorder: panic disorder, agoraphobia without history of panic disorder, generalized anxiety disorder, OCD, PTSD, specific and social phobia; any mood disorder: major depression, dysthymia, bipolar disorder I or II; any non-alcohol substance use disorder: nicotine dependence or any illegal substance use disorder. 
Table 3. Earlier onset ${ }^{a}$ of a prior mental disorder (PMD) and the risk of transition to DSM-IV alcohol use disorders (AUDs)

\begin{tabular}{|c|c|c|c|c|c|c|c|c|}
\hline & \multicolumn{4}{|c|}{ Alcohol abuse } & \multicolumn{4}{|c|}{ Alcohol dependence } \\
\hline & HR & $95 \%$ CI & $p$ & $\begin{array}{l}\text { Probability } \\
>\chi^{2}(p \text { value })^{\mathrm{b}}\end{array}$ & HR & $95 \% \mathrm{CI}$ & $p$ & $\begin{array}{l}\text { Probability } \\
>\chi^{2}(p \text { value })^{\mathrm{b}}\end{array}$ \\
\hline \multicolumn{9}{|l|}{ Model I } \\
\hline Any affective disorder ${ }^{\mathrm{g}}$ & 0.97 & $0.9-1.0$ & 0.384 & 0.79 & 0.91 & $0.8-0.9$ & 0.001 & 0.91 \\
\hline Major depression & 0.82 & $0.7-0.9$ & $<0.001$ & 0.63 & 0.83 & $0.7-0.9$ & 0.001 & 0.87 \\
\hline Dysthymia & 0.67 & $0.5-0.8$ & $<0.001$ & 0.85 & N.A. ${ }^{d}$ & & & \\
\hline Bipolar disorder & 1.16 & $0.9-1.4$ & 0.108 & 0.70 & N.A. ${ }^{\mathrm{d}}$ & & & \\
\hline Any anxiety disorderg & 0.97 & $0.9-1.0$ & 0.161 & 0.67 & 0.96 & $0.9-1.0$ & 0.164 & 0.82 \\
\hline Specific phobia & 0.90 & $0.8-0.9$ & 0.003 & 0.45 & 0.94 & $0.8-1.0$ & 0.049 & 0.72 \\
\hline Social phobia & 0.94 & $0.8-1.0$ & 0.169 & 0.87 & 1.13 & $0.9-1.4$ & 0.161 & 0.66 \\
\hline Panic attacks & 0.93 & $0.8-1.0$ & 0.143 & 0.69 & 0.85 & $0.7-1.0$ & 0.074 & 0.51 \\
\hline Somatoform disorders & 0.97 & $0.9-1.0$ & 0.191 & 0.64 & 0.97 & $0.9-1.0$ & 0.434 & 0.78 \\
\hline Any substance use disorderg & 0.93 & $0.8-1.0$ & 0.060 & 0.72 & 0.84 & $0.7-0.9$ & 0.027 & 0.70 \\
\hline Nicotine dependence & 0.94 & $0.8-1.0$ & 0.154 & 0.67 & 0.78 & $0.6-0.9$ & 0.006 & 0.83 \\
\hline Cannabis abuse $\mathrm{e}^{\mathrm{cf}}$ & 0.65 & $0.4-0.9$ & 0.019 & 0.75 & 0.78 & $0.6-1.0$ & 0.057 & 0.74 \\
\hline \multicolumn{9}{|l|}{ Model II ${ }^{\mathrm{ce}}$} \\
\hline Any affective disorder ${ }^{\mathrm{g}}$ & 0.97 & $0.9-1.0$ & 0.360 & 0.78 & 0.92 & $0.8-0.9$ & 0.008 & 0.94 \\
\hline Major depression & 0.79 & $0.6-0.9$ & 0.007 & 0.57 & 0.75 & $0.6-0.9$ & $<0.001$ & 0.79 \\
\hline Dysthymia & N.A. ${ }^{d}$ & & & & N.A. ${ }^{d}$ & & & \\
\hline Bipolar disorder & 1.23 & $0.9-1.6$ & 0.081 & 0.50 & N.A. ${ }^{\mathrm{d}}$ & & & \\
\hline Any anxiety disorderg & 0.96 & $0.9-1.0$ & 0.068 & 0.58 & 0.94 & $0.9-0.99$ & 0.027 & 0.51 \\
\hline Specific phobia & N.A. ${ }^{d}$ & & & & 0.92 & $0.8-0.9$ & 0.021 & 0.96 \\
\hline Social phobia & 0.92 & $0.8-1.0$ & 0.225 & 0.83 & N.A. ${ }^{d}$ & & & \\
\hline Panic attacks & N.A. ${ }^{\mathrm{d}}$ & & & & N.A. ${ }^{d}$ & & & \\
\hline Somatoform disorders & 0.98 & $0.9-1.0$ & 0.396 & 0.66 & 0.96 & $0.8-1.0$ & 0.348 & 0.99 \\
\hline Any substance use disorderg & 0.94 & $0.8-1.0$ & 0.082 & 0.72 & 0.83 & $0.7-0.9$ & 0.034 & 0.70 \\
\hline Nicotine dependence & 0.93 & $0.8-1.0$ & 0.151 & 0.92 & 0.76 & $0.6-0.9$ & 0.006 & 0.73 \\
\hline Cannabis abuse $\mathrm{e}^{\mathrm{cf}}$ & 0.59 & $0.3-0.9$ & 0.040 & 0.32 & 0.75 & $0.5-1.0$ & 0.093 & 0.57 \\
\hline
\end{tabular}

HR, Hazard ratio ; CI, confidence interval; N.A., not applicable.

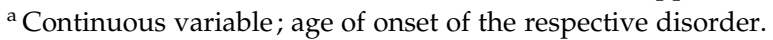

b Schoenfeld Residual Test with $p<0.05$ indicates that HRs depend on time.

${ }^{c} n=142$ excluded (unwilling to answer drug questions truthfully).

${ }^{\mathrm{d}}$ Not applicable because of insufficient statistical power.

${ }^{\mathrm{e}}$ Model I: adjusted for gender and age. Model II: additionally adjusted for substance use disorders (nicotine dependence, cannabis abuse or dependence, abuse or dependence of illegal drugs other than cannabis), any anxiety disorder (not if the covariate of interest was an anxiety disorder), and any mood disorder (not if the covariate of interest was a mood disorder). When the covariate of interest was a substance use disorder, the specific analysis was not adjusted for this particular, but for all other substance use disorders.

${ }^{\mathrm{f}}$ Hierarchy rule not applied.

g Any anxiety disorder: panic disorder, agoraphobia without history of panic disorder, generalized anxiety disorder, obsessive-compulsive disorder (OCD), post-traumatic stress disorder (PTSD), specific and social phobia; any mood disorder: major depression, dysthymia, bipolar disorder I or II; any non-alcohol substance use disorder : nicotine dependence or any illegal substance use disorder.

interaction effect HR 1.06, 95\% CI 1.006-1.12, $p=0.029$ ). Because of insufficient statistical power, these associations could not be investigated in model II. After adjustment for covariates, later age of onset of cannabis abuse was associated with a faster transition to AA (main effect HR 0.05, interaction effect HR 1.76,
$95 \%$ CI 1.17-2.66, $p=0.007)$. No other significant association with speed was found (the results on the interaction with time are available upon request). Externalizing disorders were not considered here because of lacking variance in age of onset (see Covariates section). 


\section{Discussion}

We examined, in a community sample of adolescents and young adults, whether different PMDs are associated with a higher risk and speed of transition to AUDs, whether associations with speed differed by gender and whether early onset of PMD was associated with rapid transitions. The main findings are: (1) several specific PMDs were associated with a higher risk of AUDs, but only social phobia and externalizing disorders were associated with a higher speed of transition (to AD). (2) No gender differences in associations between PMD and the speed of transition were found. (3) Early onset of several PMD was associated with higher risk but not with the speed of transition.

Several PMDs predicted AUDs. Bipolar disorders predicted AUDs after adjustment for covariates. This adds to cross-sectional evidence on the co-morbidity of bipolar disorder/mania and AD (Burns \& Teesson, 2002; Grant et al. 2004b) by showing that bipolar disorders are risk factors for AUD in adolescence and young adulthood. In accordance with the study by Buckner et al. (2008a), we found no association between major depression and $\mathrm{AD}$ after adjustment for anxiety and other disorders. A mediational relationship may exist between anxiety disorders, major depression and AUDs (Buckner et al. 2008a), as anxiety disorders typically occur earlier than mood disorders and predict these (Bittner et al. 2004; Kessler et al. 2005; Beesdo et al. 2010).

Non-alcohol substance use disorders were consistently associated with the risk of AUDs, even after adjustment for externalizing disorders. Externalizing disorders are important risk factors for substance use disorders and the existence of a shared vulnerability has been suggested (Krueger, 1999; Sung et al. 2004; McGue \& Iacono, 2008). Our results suggest that once a substance use disorder has occurred, it may contribute to the development of AUDs independent of externalizing disorders. It is of concern that nicotine dependence, which predicted AUD, is highly prevalent (cumulative incidence rate $28.5 \%$; Wittchen et al. 2008 ) in this young sample. Nicotine dependence and cannabis abuse may be proximal risk factors for AUD. This is indicated by the overlap of the incidence periods of these disorders (Wittchen et al. 2008) and leaves little time for intervention before a multiple substance use disorders status develops.

Externalizing disorders were associated with a higher risk of AUDs and a faster transition to AD, independent of all other PMDs. This adds to the consistent findings on these disorders as risk factors for AU and AUDs (Bonomo et al. 2004; King et al. 2004; McGue \& Iacono, 2008). The impulsivity observed in these disorders may foster a rapid development of excessive AU.

Specific phobia was associated with the risk of AD. Specific phobia may represent an early general vulnerability for anxiety, which may be related to AD (Brückl et al. 2007; Stinson et al. 2007; Fehm et al. 2008; Beesdo et al. 2010). However, the association was independent of other anxiety disorders and may thus be specific. In adults, specific phobia is associated with impaired functioning (Ramsawh et al. 2009), which may be associated with AD. However, high case numbers for specific phobia may have played a role here.

Social phobia was associated with a higher risk and speed of transition to AD even after adjustment for covariates. This adds to the finding that social phobia predicts AD in early adulthood independent of other mental disorders reported at about age 16 (Buckner et al. 2008a). Our adjustment took into account other PMDs, including those with incidence phases that reach into late adolescence/early adulthood, as nicotine and drug dependence (de Graaf et al. 2003; Kessler et al. 2005; Wittchen et al. 2008) and panic attacks that predict AUDs (Goodwin et al. 2004). Impaired life quality and individual functioning and the intent to relieve anxiety in social situations in social phobia (Thomas et al. 2003; Acarturk et al. 2008; Fehm et al. 2008) may contribute to instrumental AU and thus to the higher risk and speed of transition to AD. In conclusion, social phobia is an important promoter for $\mathrm{AD}$ and rapid transitions to $\mathrm{AD}$ in adolescence and young adulthood. Thus, it may be predictive of early-onset $\mathrm{AD}$, which is particularly severe (Hingson et al. 2006). Of note, these results were found with a definition of social phobia that was less strict for subjects under age 18. Some research indicates that subthreshold social phobia is associated with AD (Fehm et al. 2008).

The finding that several PMDs are associated with the risk of AUD independent of other PMDs may indicate the existence of different underlying mechanisms such as impulsivity, self-medication by use of anxiolytic alcohol effects, and cross-sensitivity or cross-tolerance for different substances.

Associations with speed of transition were few. Thus, our results confirm for several PMDs that one factor (i.e. early AU onset) is not necessarily associated with both risk and speed of transition to AUD (DeWit et al. 2000 ; Behrendt et al. 2009). Prerequisites of heavy AU (e.g. availability, social acceptance) may not be present until later adolescence (Poelen et al. 2005; van Zundert et al. 2006). This may also explain the lack of gender differences in the speed of transition in relation to PMDs. This lack of gender differences occurs in accordance with recent studies that find small or no 
gender differences in the speed of transition to AD (Wagner \& Anthony, 2007; Wittchen et al. 2008).

Nicotine dependence and cannabis use disorders were not associated with more rapid transitions to AUD, possibly because their main incidence phases overlap with those of AUD (Wittchen et al. 2008). These disorders may be proximal predictors that occur towards the end of the transition to AUD. Subjects with mood disorders may not experience the shortlived alcohol effects as a significant contribution to symptom relief, which may prevent a fast development of instrumental drinking.

Early age of onset of PMD was associated with a higher risk but not a higher speed of transition to AUD. An excess risk of AUD was found for early onset of nicotine dependence, specific phobia and cannabis abuse. In contrast to major depression per se, early major depression was associated with the risk of AUD independent of anxiety disorders. Thus, early psychopathology may be a relevant distal risk factor for AUD and warrants attention in the prevention of AUD in adolescence/early adulthood. Our results add to the finding that problem behaviors in childhood and adolescence predict AU-related problems in adulthood (Pitkänen et al. 2008). The links between early psychopathology and AUD remain to be identified. The stability of psychopathology into adolescence may play a role here (Dubow et al. 2008; Hayatbakhsh et al. 2008).

\section{Limitations}

We could not consider all PMDs of interest because of insufficient statistical power. The study covers the high-risk phase of AUD in adolescence/early adulthood but does not permit conclusions concerning AUD onset in middle/late adulthood. We used retrospective age-of-onset information that may underlie recall bias.

Future research should identify factors associated with rapid transitions to AUD and the mechanisms linking early psychopathology with AUD. Here, investigating the role of parental AU and AUD would be of interest. It would be important to investigate which characteristics of PMDs as certain symptoms or subtypes (as of specific phobia) are related to AUDs. We used non-hierarchical AA diagnosis in order to take into account all AUDs that occurred over the observed period. In future research it may be of interest to investigate cases that develop AA only.

In summary, we could show that PMDs are rarely associated with rapid transitions to AUDs in adolescence and early adulthood. However, different PMDs play an important role as risk factors for AUDs in this period.

\section{Acknowledgements}

Funding and support: this paper was prepared in the context of the project 'Community-based need evaluation II and allocation and transfer' (primary investigator: H.-U. Wittchen) of the German Addiction Research Network ASAT (Allocating Substance Abuse Treatments to Patient Heterogeneity). Contact information: email: asatkoordination@mpipsykl.mpg.de (www.asat-verbund.de). This work is part of the Early Developmental Stages of Psychopathology (EDSP) Study and is funded by the German Federal Ministry of Education and Research (BMBF), project numbers 01EB9405/6, 01EB 9901/6, EB01016200, 01EB0140 and 01EB0440. Some of the fieldwork and analyses was also supported by grants from the Deutsche Forschungsgemeinschaft (DFG) LA1148/1-1, WI2246/ 1-1, WI 709/7-1 and WI 709/8-1. The principal investigators are Dr H.-U. Wittchen and Dr R. Lieb. Core staff members of the EDSP group are: Dr K. von Sydow, Dr G. Lachner, Dr A. Perkonigg, Dr P. Schuster, Dr M. Höfler, H. Sonntag, Dr T. Brückl, E. Garczynski, Dr B. Isensee, A. Nocon, Dr C. Nelson, H. Pfister, Dr V. Reed, B. Spiegel, Dr A. Schreier, Dr U. Wunderlich, Dr P. Zimmermann, Dr K. Beesdo-Baum, Dr A. Bittner, Dr S. Behrendt and S. Knappe. Scientific advisers are Dr J. Angst (Zurich), Dr J. Margraf (Basel), Dr G. Esser (Potsdam), Dr K. Merikangas (NIMH, Bethesda), Dr R. Kessler (Harvard, Boston) and Dr J. van Os (Maastricht). The EDSP project and its family genetic supplement have been approved by the Ethics Committee of the Medical Faculty of the Technische Universitaet Dresden (no: EK-13811). All participants provided informed consent.

\section{Declaration of Interest}

Dr K. Beesdo-Baum has received speaking honoraria from Pfizer. Dr H.-U. Wittchen has received research support from Eli Lilly and Company, Novartis, Pfizer and Schering-Plough. He has also been a consultant for Eli Lilly, GlaxoSmithKline Pharmaceuticals, Hoffmann-La Roche Pharmaceuticals, Novartis, Pfizer and Wyeth, and has received speaking honoraria from Novartis, Schering-Plough, Pfizer and Wyeth.

\section{Notes}

1 Also complete information on age of onset of AU and AUD was missing in some cases (AA: $n=7, \mathrm{AD}: n=8$ ). In addition, several cases had reported onset of AUD as prior to onset of $\mathrm{AU}$ : $21(2.9 \%)$ for $\mathrm{AA}$, six $(1.9 \%)$ for $\mathrm{AD}$ (percentages refer to the total number of subjects with the respective AUD and provided age of onset information for AU and AUD). These cases had to be excluded from the Cox regression analysis. 
${ }^{2}$ Any anxiety disorder: panic disorder, agoraphobia without history of panic disorder, generalized anxiety disorder, obsessive-compulsive disorder (OCD), posttraumatic stress disorder (PTSD), specific and social phobia; any mood disorder: major depression, dysthymia, bipolar disorder I or II; any non-alcohol substance use disorder: nicotine dependence or any illegal substance use disorder.

${ }^{3}$ Because of insufficient statistical power, the analysis could not be conducted for panic disorder, generalized anxiety disorder, agoraphobia without history of panic disorder, OCD, PTSD and cannabis dependence.

\section{References}

Acarturk C, de Graaf R, van Straten A, ten Have M, Cuijpers P (2008). Social phobia and number of social fears, and their association with comorbidity, health-related quality of life and help seeking. A population-based study. Social Psychiatry and Psychiatric Epidemiology 43, 273-279.

Beesdo K, Bittner A, Pine DS, Stein MB, Höfler M, Lieb R, Wittchen H-U (2007). Incidence of social anxiety disorder and the consistent risk for secondary depression in the first three decades of life. Archives of General Psychiatry 64, 903912

Beesdo K, Pine DS, Lieb R, Wittchen H-U (2010). Incidence and risk patterns of anxiety and depressive disorders and categorization of generalized anxiety disorder. Archives of General Psychiatry 67, 47-57.

Behrendt S, Wittchen H-U, Höfler M, Lieb R, Beesdo K (2009). Transitions from first substance use to substance use disorders in adolescence: is early onset associated with a rapid escalation? Drug and Alcohol Dependence 99, 68-78.

Bittner A, Goodwin RD, Wittchen H-U, Beesdo K, Höfler M, Lieb R (2004). What characteristics of primary anxiety disorders predict subsequent major depressive disorder? Journal of Clinical Psychiatry 65, 618-626.

Blazer DG, Kessler RC, McGonagle KA, Swartz MS (1994). The prevalence and distribution of major depression in a national community sample: the National Comorbidity Survey. American Journal of Psychiatry 151, 979-986.

Bonomo YA, Bowes G, Coffey C, Carlin JB, Patton GC (2004). Teenage drinking and the onset of alcohol dependence: a cohort study over seven years. Addiction 99, 1520-1528

Brückl TM, Wittchen H-U, Höfler M, Pfister H, Schneider S, Lieb R (2007). Childhood separation anxiety and the risk of subsequent psychopathology: results from a community study. Psychotherapy and Psychosomatics 76, 47-56.

Buckner JD, Schmidt NB, Lang AR, Small JW, Schlauch RC, Lewinsohn PM (2008a). Specificity of social anxiety disorder as a risk factor for alcohol and cannabis dependence. Journal of Psychiatric Research 42, 230-239.

Buckner JD, Timpano KR, Zvolensky MJ, Sachs-Ericsson N, Schmidt NB (2008b). Implications of comorbid alcohol dependence among individuals with social anxiety disorder. Depression and Anxiety 25, 1028-1037.

Buckner JD, Turner RJ (2009). Social anxiety disorder as a risk factor for alcohol use disorders: a prospective examination of parental and peer influences. Drug and Alcohol Dependence 100, 128-137.

Burns L, Teesson M (2002). Alcohol use disorders comorbid with anxiety, depression and drug use disorders. Findings from the Australian National Survey of Mental Health and Well Being. Drug and Alcohol Dependence 68, 299-307.

Crum RM, Green KM, Storr CL, Chan Y-F, Ialongo N, Stuart EA, Anthony JC (2008a). Depressed mood in childhood and subsequent alcohol use through adolescence and young adulthood. Archives of General Psychiatry 65, 702-712.

Crum RM, Pratt LA (2001). Risk of heavy drinking and alcohol use disorders in social phobia: a prospective analysis. American Journal of Psychiatry 158, 1693-1700.

Crum RM, Storr CL, Ialongo N, Anthony JC (2008b). Is depressed mood in childhood associated with an increased risk for initiation of alcohol use during early adolescence? Addictive Behaviors 33, 24-40.

de Graaf R, Bijl RV, Spijker J, Beekman ATF, Vollebergh WAM (2003). Temporal sequencing of lifetime mood disorders in relation to comorbid anxiety and substance use disorders. Findings from the Netherlands Mental Health Survey and Incidence Study. Social Psychiatry and Psychiatric Epidemiology 38, 1-11.

DeWit DJ, Adlaf EM, Offord DR, Ogborne AC (2000). Age at first alcohol use: a risk factor for the development of alcohol disorders. American Journal of Psychiatry 157, 745750.

Dubow EF, Boxer P, Huesmann LR (2008). Childhood and adolescent predictors of early and middle adulthood alcohol use and problem drinking: the Columbia County Longitudinal Study. Addiction 103, 36-47.

Elkins IJ, McGue M, Iacono WG (2007). Prospective effects of attention-deficit/hyperactivity disorder, conduct disorder, and sex on adolescent substance use and abuse. Archives of General Psychiatry 64, 1145-1152.

Fehm L, Beesdo K, Jacobi F, Fiedler A (2008). Social anxiety disorder above and below the diagnostic threshold: prevalence, comorbidity and impairment in the general population. Social Psychiatry and Psychiatric Epidemiology 43, 257-265.

Gilman SE, Abraham HD (2001). A longitudinal study of the order of onset of alcohol dependence and major depression. Drug and Alcohol Dependence 63, 277-286.

Goodwin RD, Lieb R, Höfler M, Pfister H, Bittner A, Beesdo K, Wittchen HU (2004). Panic attack as a risk factor for severe psychopathology. American Journal of Psychiatry 161, 2207-2214.

Grant BF, Dawson DA, Stinson FS, Chou SP, Dufour MC, Pickering RP (2004a). The 12-month prevalence and trends in DSM-IV alcohol abuse and dependence: United States, 1991-1992 and 2001-2002. Drug and Alcohol Dependence 74, 223-234.

Grant BF, Hasin DS, Dawson DA (1996). The relationship between DSM-IV alcohol use disorders and DSM-IV major depression: examination of the primary-secondary distinction in a general population sample. Journal of Affective Disorders 38, 113-128.

Grant BF, Stinson FS, Dawson DA, Chou SP, Dufour MC, Compton W, Pickering RP, Kaplan K (2004b). Prevalence and co-occurrence of substance use disorders and 
independent mood and anxiety disorders. Results from the National Epidemiologic Survey on Alcohol and Related Conditions. Archives of General Psychiatry 61, 807-816.

Hammen C, Brennan PA, Keenan-Miller D, Herr NR (2008). Early onset recurrent subtype of adolescent depression: clinical and psychosocial correlates. Journal of Child Psychology and Psychiatry 49, 433-440.

Hasin DS, Stinson FS, Ogburn E, Grant BF (2007). Prevalence, correlates, disability, and comorbidity of DSMIV alcohol abuse and dependence in the United States. Results from the National Epidemiologic Survey on Alcohol and Related Conditions. Archives of General Psychiatry 64, 830-842.

Hayatbakhsh MR, McGee TR, Bor W, Najman JM, Jamrozik K, Mamun AA (2008). Child and adolescent externalizing behavior and cannabis use disorders in early adulthood: an Australian prospective birth cohort study. Addictive Behaviors 33, 422-438.

Haynes JC, Farrell M, Singleton N, Meltzer H, Araya R, Lewis G, Wiles NJ (2005). Alcohol consumption as a risk factor for anxiety and depression. Results from the longitudinal follow-up of the National Psychiatric Morbidity Survey. British Journal of Psychiatry 187, 544-551.

Hingson RW, Heeren T, Winter MR (2006). Age of alcohol-dependence onset: associations with severity of dependence and seeking treatment. Pediatrics 118, 755-763.

John U, Meyer C, Rumpf HJ, Hapke U (2004). Self-efficacy to refrain from smoking predicted by major depression and nicotine dependence. Addictive Behaviors 29, 857-866.

Keenan-Miller D, Hammen CL, Brennan PA (2007). Health outcomes related to early adolescent depression. Journal of Adolescent Health 41, 256-262.

Kendler KS, Prescott CA, Myers J, Neale MC (2003). The structure of genetic and environmental risk factors for common psychiatric and substance use disorders in men and women. Archives of General Psychiatry 60, 929-937.

Kessler RC, Berglund P, Demler O, Jin R, Merikangas KR, Walters EE (2005). Lifetime prevalence and age-of-onset distributions of DSM-IV disorders in the National Comorbidity Survey Replication. Archives of General Psychiatry 62, 593-602.

King SM, Iacono WG, McGue M (2004). Childhood externalizing and internalizing psychopathology in the prediction of early substance use. Addiction 99, 1548-1559.

Kliegel M, Ropeter A, Mackinlay R (2006). Complex prospective memory in children with ADHD. Child Neuropsychology 12, 407-419.

Krueger RF (1999). The structure of common mental disorders. Archives of General Psychiatry 56, 921-926.

Kushner MG, Sher KJ, Erickson DJ (1999). Prospective analysis of the relation between DSM-III anxiety disorders and alcohol use disorders. American Journal of Psychiatry 156, 723-732.

Lachner G, Wittchen H-U, Perkonigg A, Holly A, Schuster P, Wunderlich U, Türk D, Garczynski E, Pfister H (1998). Structure, content and reliability of the Munich Composite International Diagnostic Interview
(M-CIDI) substance use sections. European Addiction Research 4, 28-41.

Lieb R, Isensee B, von Sydow K, Wittchen H-U (2000). The Early Developmental Stages of Psychopathology Study (EDSP): a methodological update. European Addiction Research 6, 170-182.

McGue M, Iacono WG (2008). The adolescent origins of substance use disorders. International Journal of Methods in Psychiatric Research 17 (Suppl. 1), S30-S38.

Nelson CB, Wittchen H-U (1998). DSM-IV alcohol disorders in a general population sample of adolescents and young adults. Addiction 93, 1065-1077.

Pitkänen T, Kokko K, Lyyra A-L, Pulkkinen L (2008). A developmental approach to alcohol drinking behaviour in adulthood: a follow-up study from age 8 to age 42 . Addiction 103, 48-68.

Poelen EAP, Scholte RHJ, Engels RCME, Boomsma DI, Willemsen G (2005). Prevalence and trends of alcohol use and misuse among adolescents and young adults in the Netherlands from 1993 to 2000. Drug and Alcohol Dependence 79, 413-421.

Ramsawh HJ, Stein MB, Belik SL, Jacobi F, Sareen J (2009). Relationship of anxiety disorders, sleep quality, and functional impairment in a community sample. Journal of Psychiatric Research 43, 926-933.

Randall CL, Roberts JS, Del Boca FK, Carroll KM, Connors GJ, Mattson ME (1999). Telescoping of landmark events associated with drinking: a gender comparison. Journal of Studies on Alcohol 60, 252-260.

Reed V, Gander F, Pfister H, Steiger A, Sonntag H, Trenkwalder C, Sonntag A, Hundt W, Wittchen H-U (1998). To what degree does the Composite International Diagnostic Interview (CIDI) correctly identify DSM-IV disorders? Testing validity issues in a clinical sample. International Journal of Methods in Psychiatric Research 7, 142-155.

Rehm J, Taylor B, Room R (2006). Global burden of disease from alcohol, illicit drugs, and tobacco. Drug and Alcohol Review 25, 503-513.

Royall RM (1986). Model robust confidence intervals using maximum likelihood estimators. Statistical Review 54, 221-226.

Sansone RA, Sansone LA (2009). Early- versus late-onset dysthymia: a meaningful clinical distinction? Psychiatry (Edgmont) 6, 14-17.

Sihvola E, Rose RJ, Dick DM, Pulkkinen L, Marttunen M, Kaprio J (2008). Early-onset depressive disorders predict the use of addictive substances in adolescence: a prospective study of adolescent Finnish twins. Addiction 103, 2045-2053.

StataCorp (2009). Stata Statistical Software: Release 11.0. StataCorp LP: College Station, TX.

Stinson FS, Dawson DA, Chou SP, Smith A, Goldstein RB, Ruan WJ, Grant BF (2007). The epidemiology of DSM-IV specific phobia in the USA: results from the National Epidemiologic Survey on Alcohol and Related Conditions. Psychological Medicine 37, 1047-1059.

Sung M, Erkanli A, Angold A, Costello EJ (2004). Effects of age at first substance use and psychiatric comorbidity on 
the development of substance use disorders. Drug and Alcohol Dependence 75, 287-299.

Therneau T, Grambsch P (2000). Modeling Survival Data: Extending the Cox Model. Springer: New York.

Thomas SE, Randall CL, Carrigan MH (2003). Drinking to cope in socially anxious individuals: a controlled study. Alcoholism, Clinical and Experimental Research 27, 1937-1943.

van Zundert RMP, van Der Vorst H, Vermulst AA, Engels RCME (2006). Pathways to alcohol use among Dutch students in regular education and education for adolescents with behavioral problems: the role of parental alcohol use, general parenting practices, and alcohol specific parenting practices. Journal of Family Psychology 20, 456-467.

Wagner FA, Anthony JC (2002). From first drug use to drug dependence: developmental periods of risk for dependence upon marijuana, cocaine, and alcohol. Neuropsychopharmacology 26, 479-488.

Wagner FA, Anthony JC (2007). Male-female differences in the risk of progression from first use to dependence upon cannabis, cocaine and alcohol. Drug and Alcohol Dependence 86, 191-198.

Wilhelm K, Mitchell P, Slade T, Brownhill S, Andrews G (2003). Prevalence and correlates of DSM-IV major depression in an Australian national survey. Journal of Affective Disorders 75, 155-162.

Wittchen H-U, Behrendt S, Höfler M, Perkonigg A, Lieb R, Bühringer G, Beesdo K (2008). What are the high risk periods for incident substance use and transitions to abuse and dependence? Implications for early intervention and prevention. International Journal of Methods in Psychiatric Research 17, 16-29.
Wittchen H-U, Lachner G, Wunderlich U, Pfister H (1998a). Test-retest reliability of the computerized DSM-IV version of the Munich-Composite International Diagnostic Interview (M-CIDI). Social Psychiatry and Psychiatric Epidemiology 33, 568-578.

Wittchen H-U, Lieb R, Schuster P, Oldehinkel AJ (1999a). When is onset? Investigations into early developmental stages of anxiety and depressive disorders. In Childhood Onset of 'Adult' Psychopathology, Clinical and Research Advances (ed. J. L. Rapoport), pp. 259-302. American Psychiatric Press: Washington, DC.

Wittchen H-U, Nelson CB, Lachner G (1998b). Prevalence of mental disorders and psychosocial impairments in adolescents and young adults. Psychological Medicine 28, 109-126.

Wittchen H-U, Perkonigg A, Lachner G, Nelson CB (1998c). Early Developmental Stages of Psychopathology Study (EDSP): objectives and design. European Addiction Research 4, 18-27.

Wittchen H-U, Pfister H (1997). Instruction Manual for the DIA-X-Interview [in German]. Swets \& Zeitlinger: Frankfurt.

Wittchen H-U, Semmler G (1990). Composite International Diagnostic Interview (CIDI). Beltz: Weinheim.

Wittchen HU, Stein MB, Kessler RC (1999b). Social fears and social phobia in a community sample of adolescents and young adults: prevalence, risk factors and co-morbidity. Psychological Medicine 29, 309-323.

Zimmermann P, Wittchen H-U, Pfister H, Kessler RC, Lieb R (2003). Primary anxiety disorders and the development of subsequent alcohol use disorder: a 4-year community study of adolescents and young adults. Psychological Medicine 33, 1211-1222. 